\title{
Jan Karski: el testigo incómodo del Holocausto ante Roosevelt
}

Alicia Ors Ausín

Instituto Franklin-UAH 


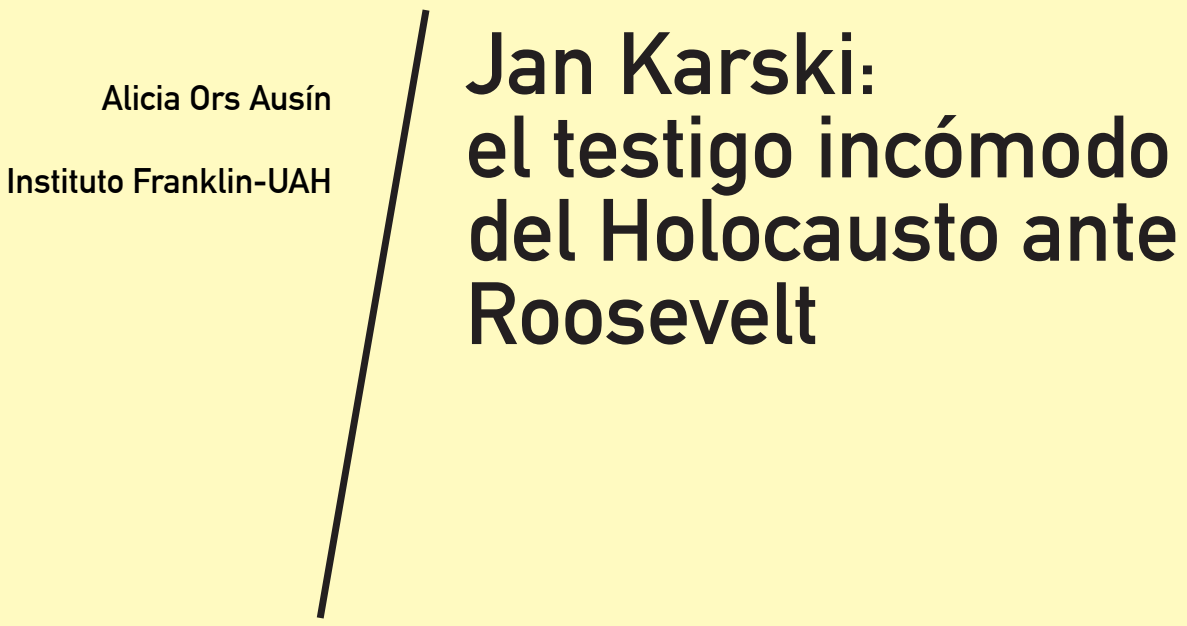

an Karski,un joven polaco fue el testigo incómodo del Holocausto.Introducido por la Resistencia de forma clandestina en el gueto de Varsovia y más tarde en el campo de tránsito de Izbica, almacenó en su imaginario personal el verdadero horror de lo que era un plan calculado por el gobierno nazi para acabar con el pueblo judío en Europa.

Palabras clave: exterminio; judíos; campos de concentración; Roosevelt; genocidio.

Alicia Ors Ausín es doctora en Estudios Norteamericanos por el Instituto Franklin-UAH, licenciada en Periodismo por la Universidad CEU-Cardenal Herrera y máster en Guión de Cine y Televisión por la UIMP. Ha trabajado en Radio Televisión Española, en Radio Televisión Valenciana, en la agencia EFE, así como en la oficina de Comunicación del presidente de la Generalitat Valenciana. También ha trabajado como asesora en diferentes proyectos de comunicación política y startups. Actualmente, es directora de comunicación en la agencia Rmedios Marketing y profesora de Teoría de la Comunicación en la Universidad CEU-Cardenal Herrera.

Ors Ausín, Alicia."Jan Karski: el testigo incómodo del Holocausto ante Roosevelt”. REDEN, vol. 2, no.1, 2020, pp. 69-92.

Recibido: 10 de diciembre de 2019. 
Entre saber, creer y ser consciente del Holocausto existe una gran diferencia. Así lo expresa el historiador y comentarista político Walter Laqueur en su libro The Terrible Secret: Suppression of the Truth about Hitler's "Final Solution".

Y sin embargo, la línea que separa los tres términos es muy fina. Lo explica Jan Karski, miembro de la Resistencia polaca en la Segunda Guerra Mundial, y posteriormente académico en la Universidad de Georgetown, en una entrevista para el documental de Claude Lanzmann Shoah. Karski es un personaje fascinante por su lucha para dar a conocer al mundo la existencia del Holocausto, pero todavía es un desconocido para el gran público. En dicha entrevista cuenta que entre 1942 y 1943 informó al gobierno polaco en el exilio y a los Aliados occidentales acerca del exterminio judío en Europa con testimonios y documentos aportados por testigos de los crímenes, pero sus noticias no tuvieron el eco que merecían. Laqueur recoge concretamente la conversación de Karski con el juez del Tribunal Supremo Americano, Felix Frankfurter:

Frankfurter told Karski that he did not believe him. When Karski protested, Frankfurter explained that he did not imply that Karski had in any way not told the truth, he simply meant that he could not believe him-there was a difference. (3)

\section{Muchas fueron las reuniones con altos cargos del gobierno estadounidense que Karski mantuvo a la vuelta de sus diferentes viajes a Europa, para manifestarles su inquietud por los asesinatos de judíos.}

El 27 de noviembre de 1944, The New York Times publica "Books of the Times", un artículo de opinión firmado por el periodista Orville Prescott, quien hace una crítica feroz a los americanos por no haber dado crédito a declaraciones de testigos, a fotografías que muestran el terror al que están siendo sometidos los judíos en Europa. Prescott pide que se lea la obra de Jan Karski, Story of a Secret State. Un libro publicado por primera vez en 1944, en el que Karski, mensajero del gobierno polaco en el exilio, cuenta de primera mano todo lo que ha visto y oído en Europa sin que nadie hiciera nada por remediarlo. Muchas fueron las reuniones con altos cargos del gobierno estadounidense que Karski mantuvo a la vuelta de sus diferentes viajes a Europa, para manifestarles su inquietud por los asesinatos de judíos. Unos encuentros que no tuvieron ningún efecto. Prescott da a conocer la figura de Karski a los lectores de su periódico, y dice de él que de no ser por la invasión nazi, muy probablemente hubiera sido un estudiante más que hubiese vivido una vida plancentera. 
Mr. Karski is a young Polish leader of the underground. Had his country not been attacked in 1939 he might have spent a leisured and pleasant life as a student of demography, the science of population. But he was called up to service as an artillery officer and soon was part of the confused retreat across southern Poland that swept so many Polish soldiers into the prison camps of the Russians without their ever having had a chance to fire their guns at the Germans. (párr. 2)

Karski fue entrevistado el 9 de febrero de 1995 para la publicación The Diary of Hannah Rosen, un compendio de artículos sobre el Holocausto basados en testimonios reales. Durante la conversación, a Karski le preguntan sobre las razones que pudieron pesar para que la administración Roosevelt no actuara antes e hiciera más para salvar a los judíos europeos. Karski respondió que los americanos no querían entrar en guerra, aunque finalmente lo hicieron porque Hitler declaró la guerra a Estados Unidos, pero hacerlo antes le hubiera acarreado a Roosevelt una importante pérdida de popularidad. No obstante, Karski reconoce que, a pesar de haberse entrevistado con él, no tuvo valor para preguntárselo. "Why didn't he extend more aid? How can I know? I couldn't ask the president, "What do you think about the Jews, what are you going to do." I couldn't. I was just a messenger".

El 21 de enero de 1945, The New York Times publica por primera vez un titular relacionado con las víctimas del nazismo que apela directamente a la sensibilidad de los americanos, "U.S Citizens Killed in Lwow by Nazis”. Del mismo modo, es la primera vez que se recogen evidencias de que los nazis quieren borrar todas las pruebas de las masacres de los judíos, porque advierten que llega su final.

Reporting the evidence of violence, torture, mass shootings and murders, both in death camps and among the civilian population of the district, the Soviet State Committee says that with the Russian advance, the Hitler Government and the German military command grew panicky and ordered measures to cover their extermination of the foreign nationals who had been in the concentration camps. (párr. 3)

\section{ENTREVISTA DE JAN KARSKI CON EL PRESIDENTE DE LOS ESTADOS UNIDOS, FRANKLIN D. ROOSEVELT}

Jan Karski explicó a Claude Lanzmann en la entrevista para el documental “Shoah”, que durante su reunión con Roosevelt el 28 de julio de 1943 le refirió el maltrato a los judíos, las deportaciones y la existencia de los campos de concentración. Una conversación que duró horas y en la que el presidente de Estados Unidos tan solo le preguntó en una ocasión por los judíos. Esta es parte de la transcripción de la conversación que tuvo lugar en ese encuentro, que ofrece la página web sobre la figura de Jan Karski, Jan Karski. Humanity's Hero. The story of Poland's wartime emissary, 


\section{Durante su reunión con}

Roosevelt el 28 de julio de

1943 le refirió el maltrato a

los judíos, las deportaciones

y la existencia de los

campos de concentración.

Una conversación que

duró horas y en la que

el presidente de Estados

Unidos tan solo le preguntó

en una ocasión por los

judíos. 
PRESIDENT: Do you cooperate with the Jews?

KARSKI: Yes. In two ways: the Jewish workers (Socialist) movement participates in the Underground resistance in close cooperation with the Polish Socialist Party. Independently, there is a special committee for the aid and protection of the Jews (Zegota, which is affiliated with the Government Delegate). There are Poles in the committee, which is run by a Jew, and which has relatively large sums of money at its disposal.

(párr. 32-33)

Después, Roosevelt se interesó por la resistencia polaca, por las condiciones de vida y por la labor de Karski, pero no utilizó la palabra "Jew" en niguna de sus preguntas posteriores. El periodista norteamericano Marvin Kalb dice que Roosevelt se debatía entre la respuesta político-militar y la indiferencia,

For example, in early 1942, he told Felix Frankfurter not to worry-that the Jews were being dispatched to Eastern Europe to build fortifications against a Soviet counter-attack. Was he lying? Or was he dissembling? Surely Roosevelt knew better. Laqueur cited an August 22, 1942, news conference, at which the President said that the report of Nazi atrocities "gives rise to the fear that [...] the barbaric and unrelenting character of the occupational regime will become more marked and maye ven lead to the extermination of certain populations." Certain populations? Was Roosevelt using code language, too? Who else but Jews? (6)

\section{DESCONOCIMIENTO E INDIFERENCIA: EL CONTEXTO CREADO POR LA PRENSA NORTEAMERICANA}

El 7 de marzo de 1943 el periódico norteamericano The New York Times, dirigido por Arthur Hays Sulzberger, publicó en la sección "Letters to The Times" (85) un artículo titulado "While We Do Nothing" firmado por la soprano británica Dorothy Moulton-Mayer. En él, esta mujer, casada con el filántropo alemán Robert Mayer y residente en Estados Unidos, reprocha a los norteamericanos vivir en cierta paz y tranquilidad mientras en Europa se suceden las atrocidades cometidas por Adolf Hitler. Literalmente, la articulista apunta que uno de los activos más fuertes de Hitler ha sido sorprender desde el principio al mundo con la incredulidad que generaron sus actos. "When he commenced his series of aggressions he profited always from the pause of petrified amazement which followed each of them". (85)

La autora apunta con sus palabras que los americanos, de una u otra manera, han escuchado o leído sobre los planes de Hitler, pero las prácticas nazis de esterilización y exterminio masivo contra ciertos grupos de ciudadanos europeos suenan tan alejadas de la realidad, tan inverosímiles, que resultan difíciles de creer como posibles en pleno siglo XX. Por eso, denuncia la soprano, las grandes potencias y organismos internacionales no saben reaccionar a tiempo y asegura que esos años de incredulidad están siendo aprovechados por Hitler para llevar a cabo su macabro plan palabra por palabra: "And now that we are awake, the horror seems too great for any mind not nurtured on Nazi principles to grasp. Yet, grasp it we must, if we are to act in time to save what may still be saved in Europe and our own honor here at home". (85) 
A través de su carta a un medio de comunicación del calado e impacto de The New York Times, esta mujer trata de abrir los ojos a la población americana sobre las atrocidades que se están cometiendo en Europa, recordando que desde el mes de julio de 1942 los asesinatos a judíos se están haciendo masivos. Incluso plantea varias preguntas al lector para hacerle más consciente de su indiferencia y, por extensión, de la indiferencia de todo el pueblo americano:

No words can compute the sum of such misery. And yet, it is your child, torn, crying from your arms, gone forever-where? Your father, your mother, old and loved, kicked into a truck and taken away-where? Your wife whom you adored [...] You yourself, respected citizen, honorable businessman, well dressed, clean, decent-beaten, bruised, covered with filth and unmentionable horrors, locked away from sight and sound of the world-where? The question now arises: What can be done? There can be no questions what any of us would do if we were confronted really with what is happening-a Jewish child running from Nazi tormentors would be helped and sheltered. The trouble is that we do not see these things. We hear of them on the radio or we view them in pictures indistinguishable from the preceding fictitious "thrills" at the movie. The cries and sobs, the torn and violated bodies, the starving babies, the desolate mothers are not real to our minds; if they were, our apathy could not continue. (85)

La autora insta al lector a la acción y es en este punto donde las buenas intenciones de MoultonMayer ponen de manifiesto su absoluto desconocimiento del Derecho Internacional. Propone el uso de herramientas diplomáticas como solución a una situación de emergencia humanitaria sin precedentes. Plantea la posibilidad de rescatar a las víctimas, proporcionarles transporte y recibirlas en Estados Unidos o en Palestina, previo permiso del país en el que se encuentran atrapadas, pero teme que la gran afluencia de refugiados judíos europeos hacia Norteamérica genere reticencias en la sociedad americana. Aún así, afirma que se trata de una dificultad fácilmente superable con una buena planificación y lanza una pregunta para la reflexión general, "Can we get hold of the surviving Jews and how can we put them into temporary shelter for a few years where they will be safe?" (85). La articulista no habla de política, habla de humanidad porque la política, dice, se está revelando lenta y excesivamente burocrática. La situación que se está viviendo en Alemania supone una realidad tan nueva y tan lejana de la imagen de una Europa civilizada, que la respuesta que plantea la autora del artículo podría calificarse de inocente y poco realista. Olvida que la salida de ciudadanos de un país para refugiarse en otro está regulada por leyes estrictas, que no menciona en su reflexión. En su lugar, la autora pide respuestas a los ciudadanos de a pie, cuando en realidad debería existir un respaldo político. Sus buenas intenciones demuestran, por lo tanto, falta de conocimiento del aparato político, a quien debería dirigirse públicamente reclamando soluciones.

Moulton-Mayer plantea la pregunta de cómo proporcionar un abrigo temporal y seguro a los judíos supervivientes, y destaca especialmente el término "temporal" porque está convencida de que los judíos afectados por el nazismo querrán volver a su país para reconstruir su vida de nuevo, una vez acabe el régimen de Adolf Hitler. La autora tiene en mente a ciudadanos alemanes, polacos, holandeses y franceses, de clase media y alta, en su mayoría profesionales, artistas, científicos, escritores con una vida y un reconocimiento social asentado durante años en sus respectivos paises. Sus prejuicios de clase la impiden ver que entre los afectados por el régimen de 
Hitler y las prácticas nazis, además de la preocupación por la suerte de los ciudadanos burgueses que animan su discurso y despiertan sus inquietudes morales, existen millones de judíos de las clases trabajadoras y del ámbito rural, muchos de ellos analfabetos, pobres y sin recursos sociales, y son precisamente estos los que tienen más dificultades para poder salir de sus países y huír del nazismo. De esta forma, la soprano avisa al lector estadounidense del destino incierto de un grupo de ciudadanos de ascendencia judía, que realmente ya intenta planificar la huida de su país, encontrando dificultades insalvables, incluso para aquellos que habían disfrutado de posiciones sociales privilegiadas.

Sugiere la autora, además, la posibilidad de sacar a los judíos de los países donde sus vidas corren peligro y enviarlos a una potencia protegida, como podría ser Suiza. Incluso, formula la opción de recoger a los judíos supervivientes con la ayuda de Cruz Roja para trasladarlos de forma segura a territorios neutrales como Inglaterra, EE. UU. o Palestina. En este punto, se dirige a la conciencia de quienes tienen el poder de hacer algo,

What of the more than 1,000 children who were to be saved out of France in the Fall and who are still there? Unfortunately, they fell victims not only to deliberately dilatory measures on the part of French authorities but also to red tape nearer home. (85)

Entre los países que cita está Palestina. Recuerda Moulton-Mayer que en 1939 el Libro Blanco dispuso que 75000 judíos pudieran entrar en Palestina en los siguientes cinco años, pero hasta ese momento tan solo 45000 lo han hecho. Una de las cuestiones prácticas que identifica es que ninguno de los países europeos posee un servicio consular para controlar pasaportes y "so if any of these unfortunately reach Palestine, they are turned back". (85) No obstante, propone que, resuelto este escollo, los judíos residentes en Palestina podrían cuidar de exiliados, e incluso, hace también un llamamiento a los cristianos en el mismo sentido,

it seems incredible that we, the so-called Christian people of the world, can calmly go about our business, eat, drink, amuse ourselves, in fact, live our normal lives, while in the world exists such misery [...] The suffering of these persecuted innocent people is another; it is removable; it should be faced and overcome. When the story of these years comes to be told in its hideous entirety we shall be face to face with the record of our inhumanity. (85)

Por último, culpabiliza al pueblo alemán por no haberse manifestado ni haber protestado ante la falta de humanidad del régimen de Hitler, ni siquiera en las universidades "when books were burned and colleagues thrust into concentration camps" (85). La articulista asegura, sin embargo, que aún no es demasiado tarde para actuar.

El hecho de que Moulton-Mayer plantee todos estos temas todavía en el fragor de la batalla de la Segunda Guerra Mundial hace que su visión sobre el conflicto subyacente, el genocidio, sea realmente útil aún hoy en día para comprender cómo es posible que tal crimen contra la humanidad se estuviese cometiendo ante los ojos impasibles de la humanidad.

Es importante recordar que la Segunda Guerra Mundial comprende una lucha global, en la que los hechos que constituyen el llamado Holocausto no pueden entenderse como crímenes de guerra, ya que dichos hechos no tuvieron lugar en los campos de batalla, ni fueron consecuencia de 


\section{La Segunda Guerra Mundial} comprende una lucha global, en la que los hechos que constituyen el llamado Holocausto no pueden entenderse como crímenes de guerra, ya que dichos hechos no tuvieron lugar en los campos de batalla, ni fueron consecuencia de acción bélica alguna, como años después se demostrará en los juicios de Nuremberg. 


\section{Los juicios de Nuremberg se} convirtieron en los primeros de

la historia por crímenes contra la humanidad en el mundo. Así se puso de manifiesto en la declaración inicial que los inauguró, y donde se dijo que el verdadero acusador en aquel tribunal era la humanidad. 
acción bélica alguna. Como años después se demostrará en los juicios de Nuremberg, los asesinatos de millones de civiles cometidos por los nazis constituyen un crimen contra la humanidad de proporciones nunca antes imaginadas y tipificado hoy en día como genocidio en el Derecho Internacional, y por tanto, una instancia jurídica por encima de las leyes de cualquier país. De esta forma, los juicios de Nuremberg se convirtieron en los primeros de la historia por crímenes contra la humanidad en el mundo. Así se puso de manifiesto en la declaración inicial que los inauguró, y donde se dijo que el verdadero acusador en aquel tribunal era la humanidad.

Tan solo partiendo del conocimiento de estas premisas somos capaces de entender por qué Jan Karski y su discurso ante Roosevelt sobre los hechos que acontecían en Europa parecía del todo inverosímil.

\section{EL HOLOCAUSTO: EL SECRETO DE LA SEGUNDA GUERRA MUNDIAL}

Según coinciden diferentes fuentes historiográficas, las causas bélicas de la Segunda Guerra Mundial son: en Occidente la invasión de Polonia por parte de las tropas alemanas, y en Oriente, la invasión japonesa de China, las colonias británicas, neerlandesas y posteriormente el ataque a Pearl Harbor.Acciones agresivas todas ellas que recibieron como respuesta la declaración de guerra de Estados Unidos contra Europa. No obstante, entre todas esas causas no figura el Holocausto porque, como ya hemos comentado anteriormente, los hechos que se engloban bajo ese término no formaban parte de la contienda.

Según explica el profesor Walter Laqueur, durante 1943 y los inicios de 1944 los asesinatos masivos a judíos, que se están produciendo por todo el territorio invadido por el ejercito alemán, no figuraban de forma destacada en los medios de comunicación de los países Aliados y neutrales, ni tampoco se mencionaban en las declaraciones oficiales de las potencias aliadas."Many American and British Jews realized the full extent of the catastrophe only during the last year of the war and many non-Jews only after the war had ended".(6)

Revela Laqueur que en enero de 1943, justo después de la declaración de los Aliados condenando las atrocidades de los Nazis contra los judíos, más de la mitad de los ciudadanos americanos entrevistados para una encuesta sobre este tema no podían creer que los Nazis estuvieran asesinando deliberadamente a los judíos. Otra investigación similar realizada a finales de 1944 mostraba que la mayoría de los americanos todavía creía que menos de 100000 judíos habían sido exterminados bajo el mandato de Adolf Hitler.Afirma el profesor Laqueur que tampoco se le dio demasiada relevancia política a estas encuestas que dejaban entrever una y otra vez "a regrettable lack of information about facts and figures in general including, for instance, the size of the population of the United States or even their home state or town".(6)

El día que se publicó la citada carta firmada por Dorothy Moulton-Mayer, el 7 de marzo de 1943, hacía ya casi un año y tres meses que se había celebrado en una zona burguesa de Berlín, Wannsee, la conferencia que debatió la "Solución final al problema judío en Europa", el 20 de enero de 1942. Con este macabro eufemismo se referían los nazis al asesinato deliberado y 
cuidadosamente planificado de millones de ciudadanos europeos, una matanza inconmensurable de seres humanos que después será conocida como genocidio de los judíos europeos. Aún así, Wannsee no marcó el inicio de aquel histórico exterminio, tan solo fue un lugar donde concretar dentro de las más altas instancias nazis, el plan que ya venía ejecutándose desde 1941 con las cámaras de gas experimentales de Auschwitz, y anteriormente con las unidades móviles en Chelmno, Treblinka, Sobibor y Belzec. Más de dos años, pues, en que las autoridades y miembros de la sociedad civil estadounidenses escuchaban atónitos las noticias intermitentes que llegaban sobre lo que estaba ocurriendo en Europa, sin dar crédito a las acciones y los métodos, que se decían, utilizados por Hitler, un líder elegido democráticamente por el pueblo alemán. Las noticias sobre las matanzas contra civiles señalaban la impunidad con que se estaba llevando a cabo un plan estratégicamente pensado para exterminar al pueblo judío asentado durante siglos en Europa, y de esta forma acabar con cualquier vestigio de su cultura.

Sobre la importancia de la conferencia de Wansee reflexiona Laqueur, apuntando que esta cita fue la ocasión en la que Adolf Eichmann convocó a los representantes de varios ministerios alemanes, cuyo apoyo le era imprescindible para acelerar la "Solución final". Fue un paso importante, dice Laqueur, pero en modo alguno fue el principio del exterminio. "In the six months preceding this conference more than half a million Jews had already been killed by the special SS units, the Einsatzgruppen, and the first extermination centre (Chelmno) already functioned".(6)

En abril de 1945, una vez liberado Auschwitz, los soldados de las filas Aliadas que participaron en la cancelación de aquella especie de prisiones diseminadas por Europa desconocían la naturaleza de los campos de concentración nazi y manifestaban su horror ante lo que iban descubriendo: ¿Eran campos de trabajos forzados? ¿Eran lugares para recluir enfermos? ¿Eran campos de prisioneros en situación de desnutrición y suciedad extremos? ¿Se trataba de espacios de tortuna y lugares de exterminio? Hay que tener en cuenta que cuando las tropas británicas entraron por primera vez en el campo de Bergen-Belsen, en abril de 1945, las actividades de exterminio sistemático habían cesado meses antes. Las unidades especiales de las SS se habían afanado, viendo el desarrollo y avance de las tropas Aliadas, en borrar las huellas del exterminio masivo destruyendo los campos y evacuando a los prisioneros a través de las conocidas como marchas de la muerte, que consistían en trasladarlos lejos de los aliados, hacia campos del interior de Alemania, para prevenir que pudieran apresarlos y contaran los asesinatos masivos. Se trataba de marchas en las que los prisioneros recorrían largas distancias bajo unas estrictas medidas de seguridad, sufriendo maltrato por parte de los guardias de las SS y soportando unas condiciones meteorológicas extremas. Muchos de los prisioneros no sobrevivieron a estos traslados y otros fueron asesinados en el trayecto, ya que los guardias tenían órdenes de disparar a todos aquellos que mostrasen síntomas de agotamiento.

Según recuerda el United States Holocaust Memorial Museum de Washington en un artículo titulado "La liberación de los campos nazis", en julio de 1944 las fuerzas soviéticas encontraron el campo de Majdanek cerca de Lublin, en Polonia, donde los alemanes intentaron esconder la evidencia del exterminio, 
Las autoridades y miembros de la sociedad civil estadounidenses escuchaban atónitos las noticias

intermitentes que llegaban sobre lo que estaba ocurriendo en Europa, sin dar crédito a las acciones y los métodos, que se decían, utilizados por Hitler, un líder elegido democráticamente por el pueblo alemán. 
El personal del campo incendió el crematorio grande, pero en la apurada operación quedaron intactas las cámaras de gas. En el verano de 1944, los soviéticos también llegaron a los campos de exterminio de Belzec, Sobibor, y Treblinka. Los alemanes habían desmontado estos campos en 1943, después que la mayoría de los judíos polacos habían sido matados (sic.). En enero de 1945, los soviéticos liberaron Auschwitz, el campo de exterminio y concentración más grande [...] Había abundante evidencia del exterminio masivo en Auschwitz. Los alemanes habían destrozado la mayoría de los depósitos en el campo, pero en los que quedaban los soviéticos encontraron las pertenencias de las victimas. (USHMM párr. 2)

Pero no en todos los campos existieron cámaras de gas. Es interesante en este punto prestar atención y analizar brevemente el sistema de campos de concentración que establecieron los nazis, ya que no en todos ellos se produjeron exterminios planificados en cámaras de gas,y algunos campos evolucionaron de ser originariamente centros de detención a convertirse en campos de la muerte.

En un artículo titulado "El sistema de los campos de concentración en profundidad" publicado por el United States Holocaust Memorial Museum, se apunta la primavera de 1933 como el momento en el que las SS establecieron el campo de concentración de Dachau, "que sirvió como modelo para un sistema de campos de concentración centralizados y en expansión" (párr. 2). En un principio se trataba de lugares de detención para recluir a opositores políticos al régimen, y con el tiempo se convirtieron en lugares "donde las personas eran encarceladas sin respeto por las normas que habitualmente se aplican al arresto y la custodia" (párr. 1). Este mismo artículo establece una tipología de campos existentes en Europa que detallo a continuación:

- Campos de trabajos forzados: donde se obligaba a los prisioneros a trabajar en condiciones infrahumanas. Kaufering/Landsberg, Flossenbürg, Dachau, Buchenwald, Breitenau o Mittelbau/Dora, en Alemania, son algunos ejemplos.

- Campos de prisioneros de guerra: donde eran recluidos los prisioneros que los alemanes realizaban durante la contienda. Niederhagen, en Alemania, o Grini, en Noruega, son algunos casos.

- Campos de tránsito: donde se realizaba la primera criba de presos, como ocurría en Theresienstadt, en la República Checa.

- Campos de concentración: es la evolución de los campos de trabajos forzados que, con el tiempo, se convirtieron en lugares donde las condiciones de trabajo y de vida eran infrahumanas. Mauthausen en Austria es un sombrío ejemplo.

- Campos de exterminio: también conocidos como campos de la muerte, de los que ya nunca más se volvía. Auschwitz-Birkenau y Chelmno, en Polonia, son algunos de los que encabezan esta lista infame.

El mapa de centros creados por los nazis para acabar con la vida de los judíos en Europa era desde un principio, y tal como se comprueba tras la liberación, una especie de puzzle desordenado de piezas aterradoras y sin sentido dentro de los parámetros del mundo civilizado. Escapaban a la moral y a la lógica de la razón de los valores que supuestamente habían alumbrado la cultura europea y, para las tropas extranjeras, carecían de toda explicación. ¿Cómo articular y dar explicación a aquello que no la tenía? 
The camps in which systematic extermination had been practised had ceased to function months earlier. In comparison with the death camps, Belsen was almost an idyllic place; there were no gas chambers in Belsen, no mass executions, death was merely by disease and starvation. But at the time it was considered the greatest possible abomination, and the luckless commanders and guards of Belsen were the first to be brought to trial; their colleagues who had been in charge of the death camps in the East were to appear in court only many years later and some would never be judged. Some had died or disappeared, others were too old or too sick, the witnesses had forgotten or died, too much time had passed. (Laqueur 2)

Este es, según recoge el United States Holocaust Memorial Museum en su archivo online, el mapa de los campos nazis en Europa durante 1943 y 1944.

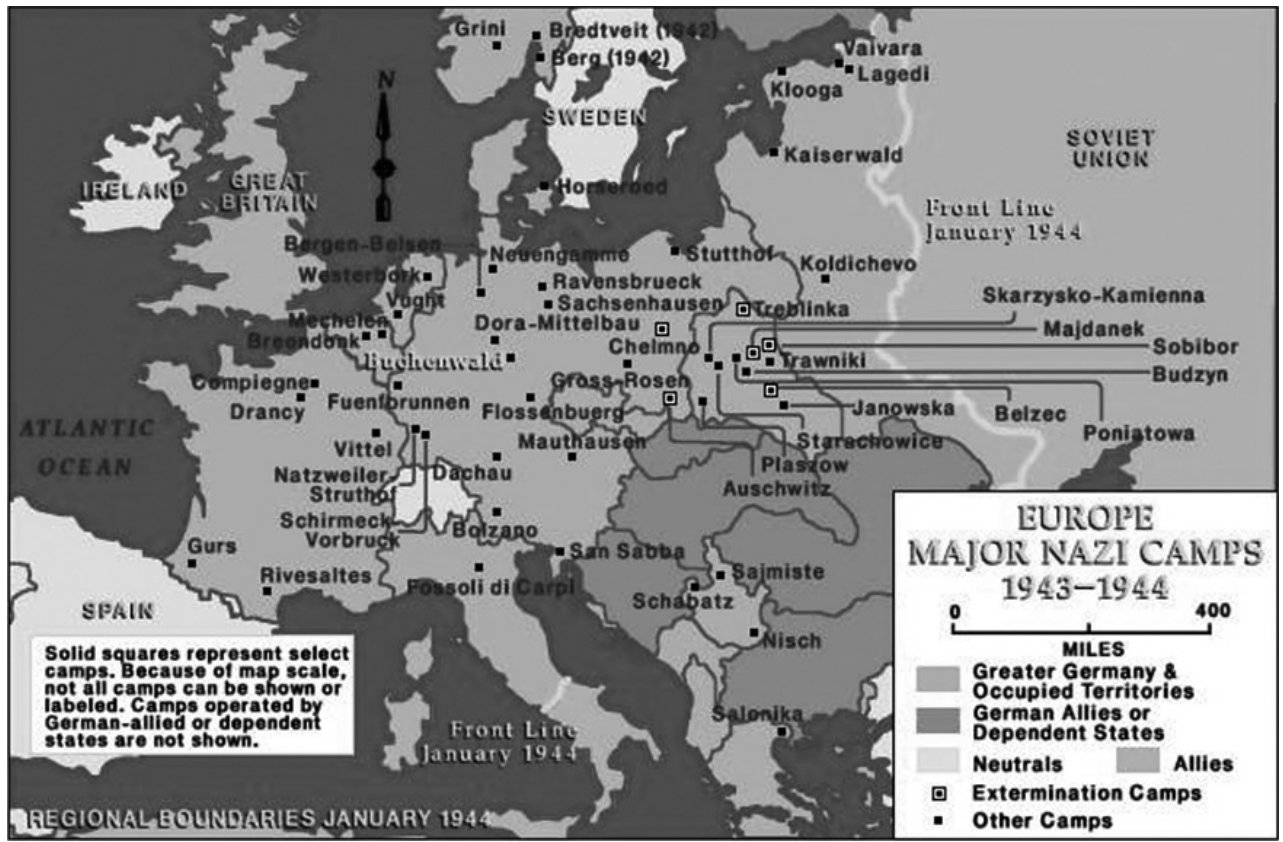

Figura 1: Mapa de los campos nazis en Europa 1943-1944 (USHMM).

Este contexto, unido al escaso y discontinuo flujo de información que llegaba a Estados Unidos en lo que se quiso entender como rumores acerca de las prácticas nazis contra los judíos, generó un vacío de comunicación al respecto. No es un tema baladí éste último,ya que los medios informativos, con sus reportajes y su labor desde el lugar de los hechos son, según un estudio de Sandra D. Melone, Georgios Terzis y Ozsel Beleli, los que alteran el entorno de comunicación y los que tienen capacidad de convertirse en supervisores de las posibles violaciones de los derechos humanos que pudieran estar cometiéndose en el escenario de la contienda, porque aportan "advertencias precoces sobre posibles intensificadores del conflicto" (3).

Unir, por tanto, todas las piezas de aquel puzzle inconexo de campos de concentración diseminados por Europa llevó mucho tiempo, y solo con tiempo se pudo observar ese mapa 
al completo y calibrar el peso de lo que acababa de ocurrir en pleno siglo XX en un escenario desarrollado y de la más sofisticada civilización como era Europa.

\section{THE NEW YORK TIMES - EL PERIÓDICO MÁS LEÍDO Y QUE MENOS USÓ LOS TÉRMINOS 'EXTERMINATION' $Y$ 'JEW'}

Entre el 20 de enero de 1942 y el 27 de enero de 1945, fecha de la liberación del campo de Auschwitz, The New York Times publicó 42 noticias que contenían los términos 'extermination' y 'Jew'.

Seguramente no fueron suficientes noticias, porque desde la primera información que se publicó con las palabras clave citadas anteriormente, el 4 de febrero de 1942 con el titular "Our Own Fifth Column Urged. Refugees from Germany Recommended for Propaganda Work", hasta que finalmente se liberó Auschwitz, pasaron poco más de tres años.

Haciendo un recorrido a través de las noticias más desgarradoras y humanas que publicó The New York Times sobre el genocidio judío con los términos citados anteriormente, encontramos historias personales, cartas, gritos de auxilio, que incrementan su crudeza y su intensidad con el paso del tiempo, y que empiezan a publicarse en 1942 como elocuentes esbozos de que algo estaba ocurriendo. Es a partir de 1943 cuando los artículos incluyen por fin la expresión "mass murders" para dar nombre a algo nuevo que ocurría paralelamente a la guerra, fuera del campo de batalla, lejos de la mirada de la comunidad internacional, y que constituía un crimen contra la humanidad.

El 4 de febrero de 1942, en la sección "Letters to the Times", The New York Times publica una carta firmada por Gunnar Leistikov titulada "Our Own Fifth Column Urged. Refugees from Germany Recommended for Propaganda Work", en la que el autor insta constantemente a las democracias a utilizar las mismas armas de las que hace uso el enemigo, para de esta manera combatirlo.Se refiere a la propaganda para hacer contrapropaganda al mensaje nazi y cita palabras de Hitler para demostrar que el canciller hablaba, desde que llegó al gobierno, de la desaparición de los judíos.

Hitler's speeches are always made to cover and influence a special situation [...] At last, he scares his fellow-countrymen: "We know fully and well that the war can end only by the extermination of the Germanic peoples or by the desappearance of Jewry from Europe. Fort he first time in history whole peoples will not be bled, but for the first time the old Judaic law of an eye for an eye and a tooth for a tooth will be applied".(párr.1)

A lo largo de 1942, este diario recoge diferentes noticias que apuntan a la supuesta comisión de crímenes en Alemania y Polonia por parte del régimen nazi, aunque todavía no se conoce la verdadera naturaleza de los mismos y las fuentes que proporcionan la información son muy pocas. En respuesta a algunas afirmaciones, se producen una serie de protestas en Nueva York de las que se hace eco The New York Times. Son movilizaciones promovidas por el American Jewish Congress, 
The Jewish Labor Committee y el B'nai Brith. El 20 de julio de 1942, este diario titula “Fight Mass Slaughter.Jewish Groups to Hold Rally in the Garden Tomorrow”,y recuerda que la condena pública contra la "mass slaughter" se llevará a cabo al día siguiente en el Madison Square Garden.

The story will be told at the meeting of how a million Jews have been murdered by the Nazis in occupied countries, how two and one-half million are being starved to death in ghettos and the remainder of the seven million under Nazi domination exposed to economic persecution and other brutalities with a view to their extermination. (párr. 2)

Posteriormente, el 17 de octubre de 1942, The New York Times publica "Poland Indicts 10 in 400,000 Deaths. They Head Roster of 3,000 War Criminals to Be Brought to Trial after Peace", y desgrana las acusaciones una a una.

1. The German Governor of Poland, Hans Frank, charged with ordering the execution of 200,000 Poles, the confiscation of Polish property, the transfer of hundreds of thousands of Polish workers to Germany, the suppression of Polish citizenship and the establishment of ghettos. (párr. 4)

Noticias como esta llevan al presidente Roosevelt a tomar la decisión de estudiar el posible nombramiento de una comisión que, junto con las Naciones Unidas, recoja y examine todas las pruebas sobre actos criminales cometidos por los nazis en los países que han ocupado. Lo anuncia este periódico el 9 de diciembre de 1942,"President Renews Pledges to Jews. He Tells Group Every Effort Will Be Made to Fix Guilt in Axis Crimes Agains Race". Se trata de una reunión de los altos representates de las comunidades judías en Estados Unidos, de la que sale un memorandum con propuestas para el presidente Roosevelt con el objetivo de que los nazis rindan cuentas por los crímenes cometidos y las deportaciones de judíos. A continuación, este artículo recoge cifras de los judíos asesinados en Alemania, Austria, Bélgica, Bohemis, Holanda, Yugoslavia, Grecia y Rumanía, cerca de dos millones en total. Los representantes de las comunidades judías hacen uso de su influencia para conseguir ser oídos por el presidente de los Estados Unidos, que les ofrece su compromiso serio de ayuda para atajar lo que está ocurriendo en Europa con los judíos.

President Roosevelt did not doubt, Rabbi Wise said, that the United Nations would be prepared, as the American Government will be, to take every step "which will end these serious crimes against the Jews and against all other civilian populations of the Hitler-ruled countries and to save those who may yet be saved." [...] At that time you will recall, the President added,"I said the American people not only sympathize with the victims of Nazi crimes but will hold the perpetrators of these crimes to strict accuntability in a day of reckoning [sic] which will surely come." (párr. 4-8)

La reunión con los altos representantes de las comunidades judías en Estados Unidos acaba con un memorandum que recopila pruebas que doten de más fuerza,si cabe, su solicitud de ayuda urgente para los judíos en Europa.

We come to you as representative of all sections of the Jewish community of the United States. Within recent months all Americans have been horrified by the verification of the reports concerning the barbarities against the inhabitants of countries overrun by Hitler's forces. To these horrors has now been added the news of Hitler's edict calling for the extermination of all Jews in the subjugated lands. (párr.11) 
Pocos días después, el 18 de diciembre de 1942, The New York Times califica de "war" al exterminio al que los judíos están siendo sometidos por los nazis: "11 Allies Condemn Nazi War On Jews. United Nations Issue Joint Declaration of Protest on "Cold-Blooded Extermination" y recuerda el comunicado de Roosevelt del 21 de agosto de ese mismo año, en el que se denuncian las persecuciones y se advierte a los responsables de que serán llevados ante los tribunales; y la del 7 de octubre del mismo año, en el que habla de crímenes de guerra.

President Roosevelt on 21 Aug. 21, 1942, issued a statement denouncing the persecutions and warning those responsible that "the time will come when they shall have to stand in courts of law in the very countries which they are now oppressing and answer for their acts." In another statement, on Oct. 7,1942, President Roosevelt advocated a United Nations Commission for the Investigation of War Crimes for meting out "just and sure punishment" to the "ringleaders responsible for the organized murder of thousands of innocent persons and the commission of atrocities which have violated every tenet of the Christian faith." (párr.14)

A continuación,el 20 de diciembre de 1942, The New York Times publica un informe de las Naciones Unidas que ya habla del exterminio a los judíos, e incluso de los métodos empleados por los nazis. "Allies Describe Outrages on Jews", reza el titular y en él se reflexiona sobre la suerte que habrán corrido cinco millones de judíos en Europa, e incluso llega a temer que hayan sido "wiped off from the face of the earth". Un objetivo para el cual, según el informe de la ONU, los nazis podrían haber redoblado sus esfuerzos.

Reports from various and unquestionable sources confirmed that Jews were being deported from their homes and sent to concentration camps in other countries (notably Poland) in everincreasing numbers and that a large proportion of them were being put to death by methods utterly foreign to any known standards of human behavior. No attempt has been made to present a complete historical record, the committee says because "that would not be possible, for many details have been shrouded in death itself-but sufficient is shown to reveal the continentwide consistency of the persecution that is now taking place. The statement thus presents but a summary of the evidence." (párr. 4)

En ese momento ya se sabe, porque así lo cita este diario en palabras de Hubert Pierlot, político belga, profesor de Derecho y presidente del Belgian Government in exile en 1939, que el tratamiento que Alemania da a los judíos es uno de los dramas más oscuros de la historia.

The report goes on to quote Hubert Pierlot, Premier of the Belgian Government in exile, who declared that Belgians felt unanimously that "Germany's treatment of the Jews is one of the darkest dramas in history." The same conditions are reported from Luxembourg and the Netherlands, as well as the Scandinavian countries in German hands-Norway and Denmark-where the Jews, though few in number, face the same fate as in the rest of the Continent. Most of Norway's Jews are said to have been sent to concentration camps in Toensberg and Poland. (párr. 10)

A partir de 1943, las noticias sobre ejecuciones masivas se suceden, alternándose con las peticiones de auxilio internacional. El 17 de enero de 1943, encontramos el siguiente titular, "Germans clearing Silesian Corridor. Area 60 Miles Wide Is Called Scene of Biggest Drive to Exterminate Poles",y el 5 de abril del mismo año "Jewish Congress Asks Aid in Europe. Chicago Division to Push 
Succor Programs for the 5,000,000 Jews There. Warning by a Senator".Ambas noticias no ocupan la portada, sino que se encuentran publicadas entre las páginas 12 y 14 de este diario.

Algo más cercana a la portada, en la página 7, publicó el 4 de junio de 1943 The New York Times la nota de suicidio de Szamul Zygielbojm, un polaco residente en Londres como consecuencia del exilio del gobierno de Polonia. En ella, según apunta el diario en un subtítulo, la víctima pide ayuda urgente para los supervivientes y denuncia la apatía de las autoridades polacas en el exilio ante lo que está ocurriendo en Europa con los judíos.Además, se desgranan los motivos de su suicidio, que él mismo justifica diciendo que su mujer y su hijo se encuentran entre las víctimas de Hitler, y que hasta el momento él había trabajado incansablemente como miembro del Polish National Council y del Jewish Socialist Party para aliviar la situación de los innumerables oprimidos, cuyas casas en los guetos fueron gradualmente "being ground out of existence" (párr.1), pero que ya no puede más.

By my death I wish to express my strongest protest against the inactivity with which the world is looking on and permitting the extermination of Jewish people. I know how human little life is worth, especially today. But as I was unable to do anything during my life, perhaps by my death I shall contribute to destroying the indifference of those who are able and should act in order to save now, maybe at the last moment, this handful of Polish Jews who are still alive from certain anihilation. (párr.12)

Esta noticia cita tres elementos importantes para ser conscientes de que sí hubo solicitud pública de auxilio por parte de las víctimas y que sí hubo denuncia social debido a la inacción de las autoridades:

1. La carta de suicidio de Szamul Zygielbojm va dirigida al presidente polaco en el exilio, Wladislaw Raczkiewicz, y al premier, Wladislaw Sikorski, dos personas públicas.

I take the liberty of addressing to you my last words, and through you the Polish Government and people, the governments and people of the Allied States and the conscience of the world. From the latest information received from Poland, it is evident that without doubt the Germans with ruthless cruelty are now murdering the few remaining Jews in Poland. Behind the walls of the ghettos the last act of a tragedy unprecedented in history is being performed. [...] I have also to state that although the Polish Government has in a high degree contributed to stirring the opinion of the world, yet it did so insufficiently, for it did nothing extraordinary enough to correspond to the magnitude of the drama now being enacted in Poland. (párr. 4)

2. Zygielbojm señala como responsables no solo a los que él llama "perpetrators", verdugos, sino también "indirectly also it weighs on the whole of humanity." (párr.6)

The responsability for the crime of murdering all the Jewish population in Poland falls in the first instance on the perpetrators, but indirectly also it weighs on the whole of humanity, the peoples and governments of the Allied States, which so far have made no effort toward a concrete action for the purpose of curtailing 
this crime. By passive observation of this murder of defenseless millons and the maltreatment of children and women, the men of those countries have become accomplices of criminals. (párr. 6)

3. Zygielbojm desvela la existencia de organizaciones clandestinas de información, formadas por judíos y miembros de la resistencia, que trabajaban incansablemente para dar a conocer la tragedia que estaba ocurriendo en Polonia.

Out of the nearly 350,000 Polish Jews and about 700,000 Jews deported to Poland from other countries, there still lived in April of this year, according to the official information of the head of the underground Bund organization sent to the United States through a delegate of the government about 300,000. And the murders are still going on incessantly. (párr. 8)

El 25 de septiembre de 1943 The New York Times publica en su página 6 la petición del secretario de Estado, Cordell Hull, para que los judíos de los países ocupados por los nazis sean considerados legalmente prisioneros de guerra, y que las Naciones Unidas admitan todos esos territorios bajo su control. "Hull Tells Labor Aid to Jews Is Aim" es el titular, y el subtítulo reza: "Notice That All Individual Nazis Will Be Prosecuted for Crimes Is Sought". En el cuerpo de la noticia, las palabras de Hull ya se refieren a la necesidad de depurar las responsabilidades en los crímenes cometidos contra la comunidad judía.

The United States therefore, must warn the German people that the United Nations have decided to establish the identity of those Germans who are responsible for the acts of savagery against the Jews and other peoples; that as each of these criminal deeds is committed it is being investigated, and evidence is relentlessly being piled up for the purposes of justice. We also appeal to our Government and to the Governments of the United Nations", the statement continued, "to admit to all territorities under their control, Jewish refugees who may succeed in escaping from the Nazi hell. (párr. 10)

Y mientras esa ayuda llega, este diario también recoge las campañas de organizaciones judías americanas, como la American Jewish Joint Distribution, para recaudar dinero destinado a ayudar a los judíos en Alemania. El 6 de diciembre de 1943, se publica en la página 17 el siguiente titular, "Jewish Aid Group to Seek $\$ 16,000,000$. Greenstein, Chief of Welfare Branch, Says Agency Needs Support in Its Work".

A continuación, durante 1944 y enero de 1945, los titulares publicados por The New York Times sí permiten atisbar ya la verdadera situación que se vive en Europa, bajo el dominio de Hitler. Desde el 18 de mayo de 1944, el titular "Savage Blow Hit Jews in Hungary. 80,000 Reported Sent to Murder Camps in Poland - Non Jews Protest in Vain", se describen con mucha concreción las atrocidades y saqueos que sufre la población judía por parte de los nazis, y cuenta que cientos de judíos prefieren el suicidio antes que caer en manos del ejército de Hitler. Apunta, además, que la gran mayoría de la población aborrece las atrocidades cometidas contra los judíos compatriotas, "most of whose ancestors lived in Hungary for centuries and played and important role in the country's social, economic and political life". (párr. 2) Pero también reconoce que Hitler tiene muchos adeptos, a los que llama oportunistas, ya que se están beneficiando de la política de confiscación y expropiación de bienes aplicada a la comunidad judía. 


\section{El 25 de septiembre de 1943}

The New York Times publica en su página 6 la petición del secretario de Estado, Cordell Hull, para que los judíos de los países ocupados por los nazis sean considerados legalmente prisioneros de guerra, y que las Naciones Unidas admitan todos esos territorios bajo su control. 
There is no doubt that this clique has acquired a fairly large number of followers, nor is it surprising that the numbers of such sympathizers are growing, especially since scores of thousands of Jewish shops, homes and businesses are being distributed to influential prospective supporters. (párr. 4)

Pocos días después, el 20 de mayo de 1944, se advierte de que si los Aliados actuasen con rapidez, todavía podrían salvarse millones de vidas, "Speed Seen Needed for Jews' Rescue. 1,500,000 in Hungary and Rumania Can Be Saved, Says Official in Palestine". Una información que se publica en la página 5 del diario y como un breve, es decir sin apenas repercusión, cuando perfectamente podría tratarse de un tema digno de ocupar un editorial del periódico, un espacio precisamente dedicado habitualmente a aconsejar a líderes políticos sobre cómo actuar ante determinadas circunstancias.

\section{La lucha que Raphael Lemkin llevaba desde hacía tiempo, el reconocimiento del crimen contra la humanidad con independencia de la nación de la que se trate.}

Tendremos que esperar al mes de noviembre del 1944, concretamente al día 26, para que The New York Times cite declaraciones de testigos directos de los asesinatos en masa, que hablan de sistematismo en las prácticas nazis. Todos estos testimonios están recogidos en un informe al que tiene acceso la War Refugee Board, y a través de la cual se conocen los detalles. El informe reflexiona, incluso, sobre la posibilidad de que la falta de crédito a las atrocidades nazis sea la causante de la tardanza en la actuación por parte de los Aliados.

In the first detailed report by a United States Government Agency offering eyewitness proof of mass murder by the Germans [...] "The board has every reason to believe that these reports present a true picture of the frightful happenings in these camps. It is making the reports public in the firm conviction that they should be read and understood by all Americans." (párr. 1)

El reportaje aclara que la información proviene de la narración de dos jóvenes judíos eslovacos que escaparon de Birkenau el pasado 7 de abril de 1944 y recoge las palabras del presidente del Hebrew Committee for National Liberation, Peter H. Bergson, que recomienda diversas acciones a las Naciones Unidas, entre ellas una declaración conjunta que proclame los asesinatos de judíos como un crímen sin fronteras, independientemente del territorio en el que se haya cometido. Una solicitud que conecta directamente con la lucha que Raphael Lemkin llevaba desde hacía tiempo, el reconocimiento del crimen contra la humanidad con independencia de la nación de la que se trate. 
He added that his committee was recommending the following action to the United Nations concerned: "That they issue a joint declaration proclaiming that crimes committed against Hebrews in Europe, irrespective of the territory on which the crime was committed or the citizenship or lack of citizenship of the victim at the time of death, be considered as a war crime and punished as such." (párr. 10)

\section{CONCLUSIONES}

Hannah Rosen recoge en "Interview with Jan Karski" sus palabras sobre la indiferencia Americana ante el Holocausto.

Era fácil para los nazis matar judíos, porque lo hicieron. Los Aliados consideraron imposible y demasiado costoso acudir en rescate de los judíos, porque no lo hicieron. Los judíos fueron abandonados por todos los Gobiernos, jerarquías eclesiásticas y sociedades, pero miles de judíos sobrevivieron porque miles de individuos en Polonia, Francia, Bélgica, Dinamarca y Holanda ayudaron a salvar judíos. Ahora todos los Gobiernos e Iglesias dicen "Intentamos ayudar a los judíos", porque están avergonzados y quieren conservar su reputación. No ayudaron, porque seis millones perecieron, pero quienes estaban en los Gobiernos y en las Iglesias sobrevivieron. Nadie hizo lo suficiente.

El 27 de febrero de 2011, el periódico El País publica un reportaje titulado "Este hombre quiso parar el Holocausto", en referencia a Jan Karski y recoge sus palabras: "Los líderes judíos lo dejaron claro: los alemanes no intentan esclavizarnos como hacen con otros pueblos, estamos siendo sistemáticamente exterminados. Esa es la diferencia [...] Creen que exageramos, que somos unos histéricos, pero millones de judíos están condenados al exterminio" (párr. 5).

Karski fue el testigo incómodo del Holocausto. Introducido por la Resistencia de forma clandestina en el gueto de Varsovia y más tarde en el campo de tránsito de Izbica, almacenó en su imaginario personal el verdadero horror de lo que era un plan calculado por el gobierno nazi para acabar con el pueblo judío en Europa. Así se lo contó a Lanzmann en el documental Shoah, "Las imágenes de lo que presencié en el campo de exterminio son, me temo, mis posesiones permanentes. Nada me gustaría más que liberar mi mente de estos recuerdos". Una entrevista plagada de silencios interpretativos, necesarios para que Karski pudiera recomponerse tras revivir segundo a segundo el horror del Holocausto, a medida que se lo iba relatando a Lanzmann.

Su memoria fotográfica fue su única herramienta para informar al Estado polaco clandestino sobre las atrocidades que se cometían en los campos, y finalmente para llegar a entrevistarse con el presidente de los Estados Unidos. Este último encuentro acaba con la idea de que los altos dirigentes políticos, y especialmente los americanos, no sabían lo que ocurría en Europa.

Karski se entrevistó con el ministro de Asuntos Exteriores británico, Anthony Eden; pero el primer ministro, Winston Churchill, no lo recibió. El presidente norteamericano Franklin D. Roosevelt sí lo hizo, aunque con una actitud indiferente. El tiempo dio la razón a Jan Karski que, como él mismo dijo a Claude Lanzmann, tuvo que vivir por siempre con esas imágenes que le atormentaban. 
Revisar con detenimiento los titulares del diario The New York Times en el periodo comprendido entre 1936 y enero de 1945, con los filtros "extermination" y “Jew”, arroja resultados suficientes como para afirmar que no solo Jan Karski sabía lo suficiente. La prensa sabía lo suficiente, aunque no supo valorar el calado de esas informaciones. Karski se convirtió en el testigo incómodo del Holocausto ante, quizá, el hombre más poderoso en cualquier contienda: el presidente de los Estados Unidos.

Jan Karksi falleció en el año 2000 en Washington y la Universidad de Georgetown, de la que era académico, le dedicó una estatua en sus jardines donde reza lo siguiente,

Jan Karski (Jan Kozielewski), 1914-2000, mensajero del pueblo polaco ante su gobierno en el exilio, mensajero del pueblo judío ante el mundo, el hombre que alertó sobre la aniquilación del pueblo judío cuando aún había tiempo para detenerla. Nombrado por el Estado de Israel Justo entre las Naciones, héroe del pueblo polaco, profesor en la Universidad de Georgetown (1952-1992), un hombre noble que caminó entre nosotros y nos hizo mejores con su presencia, un hombre justo.

\section{Referencias}

Breitman, Richard. Official Secrets. What the Nazis Planned, What the British and Americans Knew. Penguin Books, 1998.

Karski,Jan. Historia de un estado clandestino. Acantilado, 2011.

Lanzmann, Claude, dir. Shoah. New Yorker Films, 1985.

Laqueur, Walter. The Terrible Secret: Suppression of the Truth about Hitler's "Final Solution". Routledge, 1980.

Leff, Laurel. Buried by The Times. The Holocaust and the America's most important newspaper. Cambridge University Press, 2005.

Levi, Neil; Rothberg, Michael. The Holocaust: Theoretical Readings. Edinburgh University Press, 2003.

Lipstadt, Deborah. Beyond Belief: The American Press and the Coming of the Holocaust 1933 1945. Free Press, 1986.

Luzán, Julia. “Este hombre quiso parar el Holocausto". El País. 27 Feb. 2011.

Melone, Sandra D. et al. Utilización de los medios de comunicación para la transformación de conflictos: la experiencia del Common Ground. Berghof- Foundation.

Rodríguez Andrés, Roberto y Teresa Sádaba Garraza. "Periodistas ante conflictos. El papel de losmedios de comunicación en situaciones de crisis". Universidad de Navarra, SA, 1999.

Rosen, Hannah. "Interview with Jan Karski”. Remember Org. A People's History of the Holocaust \& Genocide. 9 Feb. 1995.

USHMM. “La legislación anti-judía en la Alemania de la preguerra”. United States Holocaust Memorial Museum, Washington, DC.

--- “La liberación de los campos nazis". United States Holocaust Memorial Museum, Washington, DC. 